\title{
Modified coaxial wire method for measurement of transfer impedance of beam position monitors
}

\author{
Mukesh Kumar, ${ }^{1,2,{ }^{*}}$ L. K. Babbar, ${ }^{1}$ R. K. Deo, ${ }^{3}$ T. A. Puntambekar, ${ }^{1}$ and V. K. Senecha ${ }^{2,4}$ \\ ${ }^{1}$ Beam Diagnostics and Coolant Systems Division, Raja Ramanna Centre for Advanced Technology, \\ Indore 452013 M.P., India \\ ${ }^{2}$ Homi Bhabha National Institute, Training School Complex, Anushakti Nagar, Mumbai 400 094, India \\ ${ }^{3}$ High Power RF Laboratory, RF System Division, Raja Ramanna Centre for Advanced Technology, \\ Indore, 452013 M.P., India \\ ${ }^{4}$ Proton Linac Development Division, Raja Ramanna Centre for Advanced Technology, \\ Indore, 452013 M.P., India
}

(Received 29 September 2017; published 8 May 2018)

\begin{abstract}
The transfer impedance is a very important parameter of a beam position monitor (BPM) which relates its output signal with the beam current. The coaxial wire method is a standard technique to measure transfer impedance of the BPM. The conventional coaxial wire method requires impedance matching between coaxial wire and external circuits (vector network analyzer and associated cables). This paper presents a modified coaxial wire method for bench measurement of the transfer impedance of capacitive pickups like button electrodes and shoe box BPMs. Unlike the conventional coaxial wire method, in the modified coaxial wire method no impedance matching elements have been used between the device under test and the external circuit. The effect of impedance mismatch has been solved mathematically and a new expression of transfer impedance has been derived. The proposed method is verified through simulation of a button electrode BPM using CST STUDIO SUITE. The new method is also applied to measure transfer impedance of a button electrode BPM developed for insertion devices of Indus- 2 and the results are also compared with its simulations. Close agreement between measured and simulation results suggests that the modified coaxial wire setup can be exploited for the measurement of transfer impedance of capacitive BPMs like button electrodes and shoe box BPM.
\end{abstract}

DOI: 10.1103/PhysRevAccelBeams.21.052801

\section{INTRODUCTION}

In particle accelerators, a beam position monitor (BPM) provides position of charged particle beam for various applications like closed orbit measurement, fast orbit feedback system, slow orbit feedback system, coupled bunch instability feedback system etc. The transfer impedance of a BPM is a very important parameter and it relates its output signal with the beam current. Measurement of transfer impedance of a BPM plays a very important role in its development.

The electromagnetic field distribution of a charged particle moving with relativistic velocity inside a conducting vacuum chamber approximates to the field distribution of TEM mode inside a coaxial transmission line.

\footnotetext{
*Corresponding author. mukeshk@rrcat.gov.in

Published by the American Physical Society under the terms of the Creative Commons Attribution 4.0 International license. Further distribution of this work must maintain attribution to the author(s) and the published article's title, journal citation, and DOI.
}

Therefore, the coaxial wire method is a widely used technique for the bench measurement of beam coupling and transfer impedances of various components in particle accelerators. The coaxial wire method was proposed in the early seventies by Sands and Rees to measure energy loss of a stored beam in a cavity [1]. Since then a large number of people have used the coaxial wire method, in different forms, to measure beam coupling impedance of various components [2-9]. Generally, the characteristic impedance of the coaxial structure formed by wire and the device under test (DUT) is much higher than the impedance of the external circuit (generator and cables). In order to simulate particle beam realistically and avoid trapped modes, the impedance of the external circuit should be matched to the characteristic impedance of the coaxial structure. The most common method of impedance matching is the use of electrical network or tapered structures (Fig. 1).

The coaxial wire method is mainly used for the measurement of beam coupling impedance but this technique has also been used to measure transfer impedance of various devices like button electrode BPM and striplines. To measure transfer impedance of the BPM, the coaxial structure is connected to the vector network analyzer 

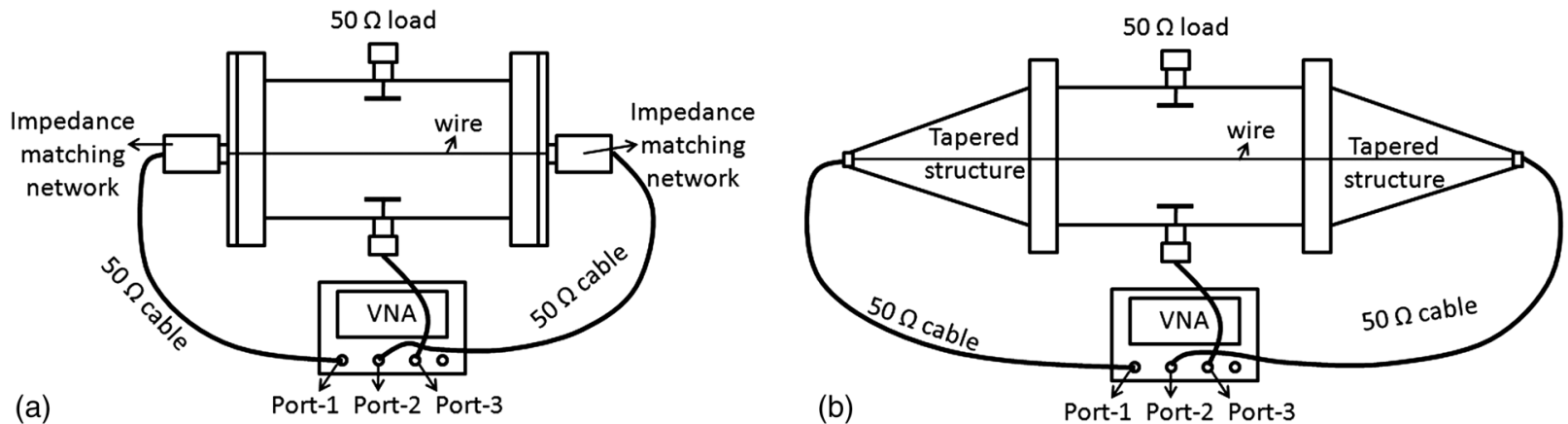

FIG. 1. Schematic setup of the conventional wire method. Impedance matching using electrical network (a) and tapered mechanical structure (b).

(VNA) as shown in Fig. 1 and transfer impedance ' $Z_{T}$ ' is calculated using Eq. (1) [10-12]:

$$
Z_{T}(\omega)=\frac{S_{31}(\omega)}{S_{21}(\omega)} \sqrt{Z_{0} Z_{w}}
$$

In Eq. (1), $S_{31}$ and $S_{21}$ are S-parameters from port-1 to port-3 and port-2 respectively, $Z_{0}$ is the characteristic impedance of the cables between VNA and BPM and $Z_{w}$ is characteristic impedance of the coaxial structure.

Measurement of the transfer impedance, using the standard (conventional) coaxial wire method, requires impedance matching between DUT and the external circuit. The implementation of impedance matching is a practically tedious task. Moreover, if characteristics impedance of the coaxial structure changes, one has to redesign/replace existing impedance matching elements accordingly. In this paper we are presenting a modified coaxial wire method for the bench measurement of the transfer impedance of capacitive BPMs like button electrodes BPM, shoe box BPM etc.

In the modified coaxial wire method, the impedance matching elements have been eliminated. The elimination of impedance matching elements results in infinite reflections inside the coaxial structure. Therefore, conventional expression of transfer impedance [Eq. (1)] is not valid in the modified coaxial wire method. Considering these reflections, a new expression for the calculation of transfer impedance of a pickup electrode has been derived. The new expression is derived using the principle of superposition of the signal induced on the pickup electrode due to infinite reflections of wire current inside DUT.

To validate the proposed (modified) coaxial wire method, the transfer impedance of a button electrode BPM is calculated by simulating it with particle beam, the conventional wire method and the modified coaxial wire method using CST STUDIO SUITE [13]. The simulation results of the modified coaxial wire method are in very good agreement with the results of the conventional wire method and particle beam based simulation of the BPM even at trapped modes. The proposed method is also applied for bench measurement of the transfer impedance of a button electrode BPM called insertion device beam position indicator (IDBPI) of Indus-2 [14-16].

This paper presents the modified coaxial wire method and derivation of new expressions of transfer impedance for capacitive pickups like button electrodes and shoe box BPMs. This paper also presents validation of the modified coaxial wire method through simulation performed using CST STUDIO SUITE and practical implementation of the modified coaxial wire method to measure transfer impedance of a button electrode BPM designed for Indus- 2 .

\section{MODIFIED COAXIAL WIRE METHOD}

The schematic setup of the modified coaxial wire method is shown in Fig. 2. Two metallic plates hold electrical feedthroughs at the entry and exit of the DUT. A wire is stretched between central pins of the electrical feedthroughs to form the coaxial structure. The VNA is directly connected to one end of the coaxial wire without using impedance matching elements. The other end of the wire is either terminated with an arbitrary load or electrically shorted at the center of the metallic plate without using electrical feedthrough. In the second case (shorted end of the wire), no electrical feedthrough is required at the second

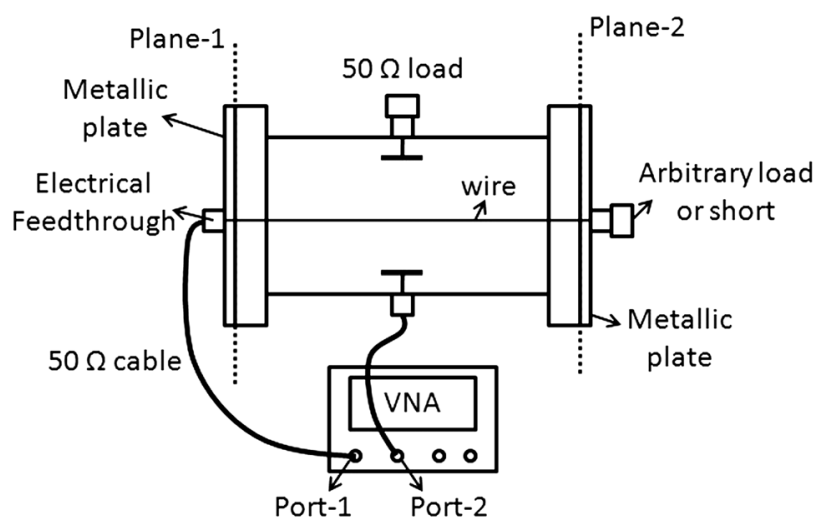

FIG. 2. Schematic of general setup of the modified coaxial wire method. 
plate, thus, practical implementation of the setup is quite easy.

\section{A. Expression for transfer impedance in the modified setup}

Let the forward voltage ' $V$ in' generated at port-1 of the VNA be

$$
V_{\text {in }}(\omega, t)=V_{0} e^{i \omega t}
$$

The voltage signal ' $V_{\text {in }}$ ' is carried to the coaxial wire using cables having characteristic impedance $Z_{0}$. Therefore the forward current ' $I$ in' generated at port-1 is

$$
I_{\text {in }}(\omega, t)=\frac{V_{0} e^{i \omega t}}{Z_{0}} .
$$

This current [Eq. (3)] travels up to the coaxial wire and undergoes partial transmission and reflection at plane- 1 . The current transmitted to the coaxial structure is given by Eq. (4)

$$
I_{1}(\omega, t)=\frac{V_{0} e^{i\left(\omega t+\phi_{0}\right)}}{Z_{0}}\left[\frac{2 Z_{w}}{Z_{0}+Z_{w}}\right]\left[\frac{Z_{0}}{Z_{w}}\right]
$$

Here $I_{1}$ is current entering the coaxial structure, $\varphi_{0}$ is phase difference between $I_{\text {in }}$ and $I_{1}$ at plane-1, $Z_{w}$ is characteristic impedance of the coaxial structure formed by wire and the device. Writing

$$
\left[\frac{2 V_{0}}{Z_{0}+Z_{w}}\right]=I_{0},
$$

Eq. (4) can be written as

$$
I_{1}(\omega, t)=I_{0} e^{i\left(\omega t+\phi_{0}\right)} .
$$

Inside the coaxial structure, the current $I_{1}$ undergoes multiple reflections at plane-1 and plane-2 (Fig. 2). Each reflected component of the current flowing through the wire generates voltage at the pickup electrodes. If $Z_{T}(\omega)=$ $\left|Z_{T}(\omega)\right| e^{i \Delta \phi}$ is transfer impedance of the pickup electrode, the net voltage ' $V_{\text {pick }}$ ' induced at the pickup electrode can be written as

$$
\begin{aligned}
V_{\text {pick }}(\omega, t)= & I_{0} e^{i\left(\omega t+\phi_{0}\right)}\left|Z_{T}(\omega)\right| e^{i \Delta \phi}+I_{0} e^{i\left(\omega t+\phi_{0}\right)}\left|\rho_{2}\right| e^{i \phi_{2}} e^{i \frac{\omega(L+2 \Delta L)}{c}}\left|Z_{T}(\omega)\right| e^{i \Delta \phi}+I_{0} e^{i\left(\omega t+\phi_{0}\right)}\left|\rho_{2}\right| e^{i \phi_{2}}\left|\rho_{1}\right| e^{i \phi_{1}} e^{i \frac{\omega(2 L)}{c}}\left|Z_{T}(\omega)\right| e^{i \Delta \phi} \\
& +I_{0} e^{i\left(\omega t+\phi_{0}\right)}\left[\left|\rho_{2}\right| e^{i \phi_{2}}\right]^{2}\left|\rho_{1}\right| e^{i \phi_{1}} e^{i \frac{(2 L)}{c}} e^{i \frac{\omega(L+2 \Delta L)}{c}}\left|Z_{T}(\omega)\right| e^{i \Delta \phi}+I_{0} e^{i\left(\omega t+\phi_{0}\right)}\left[\left|\rho_{2}\right| e^{i \phi_{2}}\right]^{2}\left[\left|\rho_{1}\right| e^{i \phi_{1}}\right]^{2}\left[e^{i \frac{\omega(2 L)}{c}}\right]^{2}\left|Z_{T}(\omega)\right| e^{i \Delta \phi \ldots} \\
& +\inf .
\end{aligned}
$$

In Eq. (7), $\left|\rho_{2}\right| e^{i \varphi 2}$ is the reflection coefficient at plane-2; $\left|\rho_{1}\right| e^{i \varphi 1}$ is the reflection coefficient at plane-1; $L$ is the length of DUT and $\Delta L$ is the distance of the pickup electrode from the center of the DUT towards plane-1; $\frac{\omega(L+2 \Delta L)}{c}=$ phase difference occurring due to distance $(L+2 \Delta L)$ traveled by the current/wave from the pickup electrode to plane-2 and back to the pickup electrode; $\frac{\omega(2 L)}{c}=$ phase difference occurring due to distance $[(L+2 \Delta L)+(L-2 \Delta L)=2 L]$ traveled by the current/wave in one complete reflection between plane-1 and plane-2.

Taking/adding alternate terms we get

$$
\begin{aligned}
V_{\text {pick }}(\omega, t)= & I_{0}\left|Z_{T}(\omega)\right| e^{i\left(\omega t+\phi_{0}\right)} e^{i \Delta \phi} \sum_{k=0}^{\infty}\left[\left|\rho_{2}\right| e^{i \phi_{2}}\left|\rho_{1}\right| e^{i \phi_{1}} e^{i \frac{\omega(2 L)}{c}}\right]^{k} \\
& +I_{0}\left|Z_{T}(\omega)\right| e^{i\left(\omega t+\phi_{0}\right)} e^{i \Delta \phi} \sum_{k=0}^{\infty}\left[\left|\rho_{2}\right| e^{i \phi_{2}}\right]^{k+1}\left[\left|\rho_{1}\right| e^{i \phi_{1}} e^{i\left(\frac{\omega(2 L)}{c}\right]^{k}} e^{i \frac{\omega(L+2 \Delta L)}{c}}\right. \\
& \Rightarrow V_{\text {pick }}(\omega, t)=I_{0}\left|Z_{T}(\omega)\right| e^{i\left(\omega t+\phi_{0}+i \Delta \phi\right)}\left[\frac{1}{1-\left|\rho_{1}\right|\left|\rho_{2}\right| e^{i\left(2 \frac{\omega L}{c}+\phi_{1}+\phi_{2}\right)}}+\frac{\left|\rho_{2}\right| e^{i\left(\frac{\omega(L+2 \Delta L)}{c}+\phi_{2}\right)}}{\left.1-\left|\rho_{1}\right|\left|\rho_{2}\right| e^{i\left(2 \frac{\omega L}{c}+\phi_{1}+\phi_{2}\right)}\right]} .\right.
\end{aligned}
$$

Let

$\omega t+\varphi_{0}+\Delta \varphi=\alpha$

$2 \omega L / c+\varphi_{1}+\varphi_{2}=\beta$

$\omega(L+2 \Delta L) / c+\varphi_{2}=\gamma$.

Equation (9) can be rewritten as

$$
V_{\text {pick }}(\omega, t)=I_{0}\left|Z_{T}(\omega)\right|\left[\frac{e^{i(\alpha)}}{1-\left|\rho_{1}\right|\left|\rho_{2}\right| e^{i(\beta)}}+\frac{\left|\rho_{2}\right| e^{i(\alpha+\gamma)}}{1-\left|\rho_{1}\right|\left|\rho_{2}\right| e^{i(\beta)}}\right]
$$




$$
\Rightarrow\left|V_{\text {pick }}(\omega, t)\right|=\frac{I_{0}\left|Z_{T}(\omega)\right| \sqrt{1+\left|\rho_{2}\right|^{2}+2\left|\rho_{2}\right| \cos (\gamma)}}{\sqrt{1+\left(\left|\rho_{1}\right|\left|\rho_{2}\right|\right)^{2}-2\left|\rho_{1}\right|\left|\rho_{2}\right| \cos (\beta)}} .
$$

Using Eq. (5) in Eq. (11) we get

$$
\frac{\left|V_{\text {pick }}(\omega, t)\right|}{V_{0}}=\frac{2\left|Z_{T}(\omega)\right| \sqrt{1+\left|\rho_{2}\right|^{2}+2\left|\rho_{2}\right| \cos (\gamma)}}{\left(Z_{0}+Z_{w}\right) \sqrt{1+\left(\left|\rho_{1}\right|\left|\rho_{2}\right|\right)^{2}-2\left|\rho_{1}\right|\left|\rho_{2}\right| \cos (\beta)}} .
$$

Using

$$
\frac{\left|V_{\text {pick }}(\omega, t)\right|}{V_{0}}=\left|S_{\text {pick, } 1}(\omega)\right|
$$

we get

$$
\left|S_{\text {pick }, 1}(\omega)\right|=\frac{2\left|Z_{T}(\omega)\right| \sqrt{1+\left|\rho_{2}\right|^{2}+2\left|\rho_{2}\right| \cos (\gamma)}}{\left(Z_{0}+Z_{w}\right) \sqrt{1+\left(\left|\rho_{1}\right|\left|\rho_{2}\right|\right)^{2}-2\left|\rho_{1}\right|\left|\rho_{2}\right| \cos (\beta)}} .
$$

It gives

$$
\left|Z_{T}(\omega)\right|=\frac{\left|S_{\text {pick }, 1}(\omega)\right|\left(Z_{0}+Z_{w}\right) \sqrt{1+\left(\left|\rho_{1}\right|\left|\rho_{2}\right|\right)^{2}-2\left|\rho_{1}\right|\left|\rho_{2}\right| \cos (\beta)}}{2 \sqrt{1+\left|\rho_{2}\right|^{2}+2\left|\rho_{2}\right| \cos (\gamma)}}
$$

Equation (15) gives transfer impedance of the pickup electrode in terms of S-parameter $\left(S_{\text {pick, } 1}\right)$ from wire to pickup electrode. Equation (15) is valid for any arbitrary load at downstream port (plane-2) of coaxial wire. Two special cases in which downstream port is terminated with $50 \Omega$ and electrically shorted are given below.

Case 1.- The downstream port of the coaxial wire is terminated with $50 \Omega$ load.

When the downstream port of the coaxial wire structure is terminated with $50 \Omega$ load (equal to the impedance of VNA and cables) we get $\left|\rho_{1}\right|=\left|\rho_{2}\right|$ and $\varphi_{1}=\varphi_{2}$. Using $\left|\rho_{1}\right|=\left|\rho_{2}\right|=|\rho|$, Eq. (15) can be written as

$$
\left|Z_{T}(\omega)\right|=\frac{\left|S_{\text {pick }, 1}(\omega)\right|\left(Z_{0}+Z_{w}\right) \sqrt{1+|\rho|^{4}-2|\rho|^{2} \cos (\beta)}}{2 \sqrt{1+|\rho|^{2}+2|\rho| \cos (\gamma)}} .
$$

Generally, the characteristic impedance of the coaxial structure formed by wire and the BPM is greater than the impedance of VNA. Therefore, using $\varphi_{1}=\varphi_{2}=\pi$ we get $\beta=2 \omega L / c+2 \pi$ and $\gamma=\omega(L+2 \Delta L) / c+\pi$. Thus, Eq. (16) becomes

$$
\left|Z_{T}(\omega)\right|=\frac{\left|S_{\text {pick, } 1}(\omega)\right|\left(Z_{0}+Z_{w}\right) \sqrt{1+|\rho|^{4}-2|\rho|^{2} \cos \left(\frac{2 \omega L}{c}\right)}}{2 \sqrt{1+|\rho|^{2}-2|\rho| \cos \left(\frac{\omega(L+2 \Delta L)}{c}\right)}} .
$$

If $\Delta L=0$, Eq. (17) reduces to

$$
\left|Z_{T}(\omega)\right|=\frac{\left|S_{\text {pick, } 1}(\omega)\right|\left(Z_{0}+Z_{w}\right) \sqrt{1+|\rho|^{2}+2|\rho| \cos \left(\frac{\omega L}{c}\right)}}{2} .
$$

Case 2.-The downstream port of the coaxial wire is shorted with metallic plate.

When the downstream port of the coaxial wire is shorted we get $\left|\rho_{2}\right|=1$. Using $\left|\rho_{2}\right|=1,\left|\rho_{1}\right|=|\rho|$ and $\varphi_{1}=\varphi_{2}=$ $\pi$ (in $\beta$ and $\gamma$ ), Eq. (15) can be written as

Simplifying Eq. (19) we get

$$
\left|Z_{T}(\omega)\right|=\frac{\left|S_{\text {pick, } 1}(\omega)\right|\left(Z_{0}+Z_{w}\right) \sqrt{1+(|\rho|)^{2}-2|\rho| \cos \left(\frac{2 \omega L}{c}\right)}}{2 \sqrt{2\left[1-\cos \left(\frac{\omega(L+2 \Delta L)}{c}\right)\right]}} .
$$

$$
\left|Z_{T}(\omega)\right|=\frac{\left|S_{\text {pick }, 1}(\omega)\right|\left(Z_{0}+Z_{w}\right) \sqrt{1+(|\rho|)^{2}-2|\rho| \cos \left(\frac{2 \omega L}{c}\right)}}{4 \sin \left(\frac{\omega(L+2 \Delta L)}{2 c}\right)} .
$$

Equations (17), (18) and (20) give transfer impedance for special cases of $50 \Omega$ and shorted termination of coaxial wire respectively. 


\section{VALIDATION OF MODIFIED COAXIAL WIRE METHOD}

To validate the modified coaxial wire method, a general button electrodes BPM has been taken as a test BPM. Important geometric parameters of the test BPM are given in Table I. The transfer impedance of the test BPM is calculated through simulation of the BPM with particle beam, the conventional wire method and the modified coaxial wire method using CST STUDIO SUITE. The results obtained through these methods are compared with each other to test the validity of the modified coaxial wire method.

\section{A. Simulation with particle beam}

The transfer impedance of the test BPM is calculated from the button (BPM) voltage using wakefield solver as explained in Refs. [14,17]. The test BPM is modeled in CST PARTICLE STUDIO and an electron beam having Gaussian longitudinal distribution is defined to travel along the axis of the BPM (Fig. 3). Wakefield solver simulates interaction of button electrodes with the electromagnetic field associated with the beam and gives voltage signals induced at the button electrodes. The root-mean-square (rms) length of the beam is taken to be $30 \mathrm{~mm}$ for the simulation. Fourier transforms of the voltage signal and beam current are shown in Figs. 4 and 5, respectively. The transfer impedance is calculated as the ratio of button signal to the beam current.

\section{B. Simulation of test BPM using the conventional coaxial wire method}

The transfer impedance of test BPM is also calculated by simulating it using the conventional coaxial wire method as explained in Ref. [11]. The simulation model of the conventional coaxial wire method for the test BPM is shown in Fig. 6. The radius of the wire taken along the axis of the BPM is $0.5 \mathrm{~mm}$. Two waveguide ports (port-1 and port-2) are defined at the entry and exit of the coaxial structure. These ports act as matched source and load at the upstream and downstream of the coaxial structure, respectively. Four waveguide ports (port-3 to port-6) are also defined at the output ports of the pickup electrodes to simulate $50 \Omega$ load. The results are discussed in sub Sec. III D.

TABLE I. Geometric parameters of the test BPM.

\begin{tabular}{llc}
\hline \hline Serial No. & \multicolumn{1}{c}{ Parameter } & Value \\
\hline 1 & Radius of button electrode & $5 \mathrm{~mm}$ \\
2 & Radius of beam pipe & $10 \mathrm{~mm}$ \\
3 & Annular gap between button & $0.5 \mathrm{~mm}$ \\
& $\quad$ and beam pipe & \\
4 & Thickness of button electrode & $3 \mathrm{~mm}$ \\
5 & Length of the BPM & $100 \mathrm{~mm}$ \\
\hline \hline
\end{tabular}

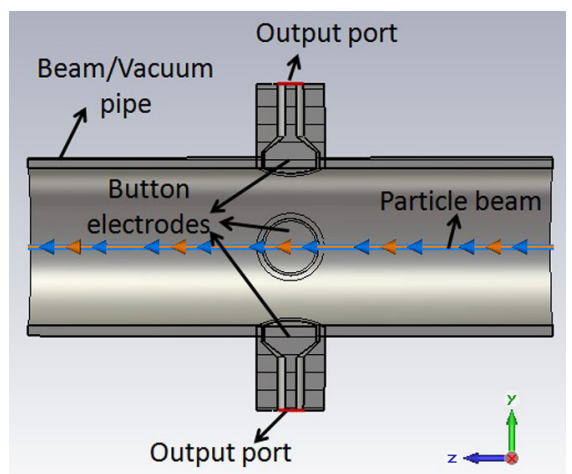

FIG. 3. CST model for the simulation of the test BPM with particle beam.

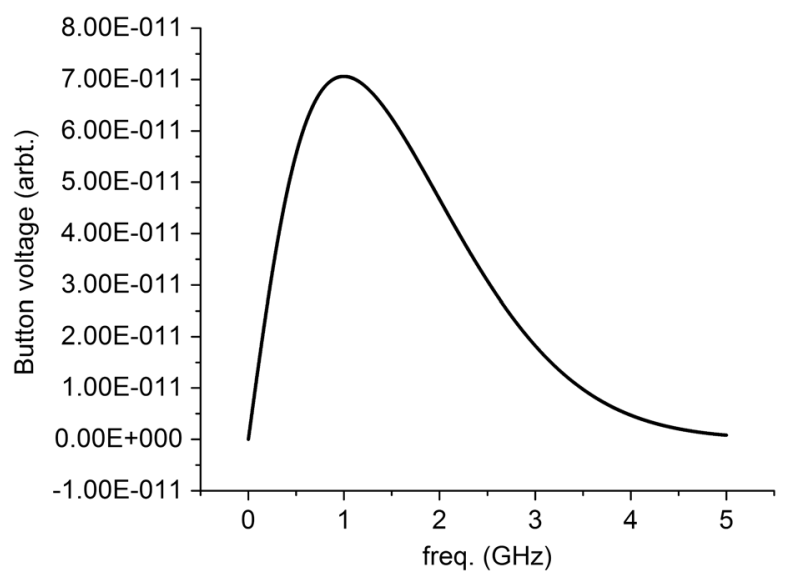

FIG. 4. Fourier transform of the voltage signal induced at the button electrode of the test BPM obtained through simulation with particle beam.

\section{Simulation of the test BPM using the modified coaxial wire method}

Simulation models of modified coaxial wire method, used to calculate transfer impedance of test BPM, are

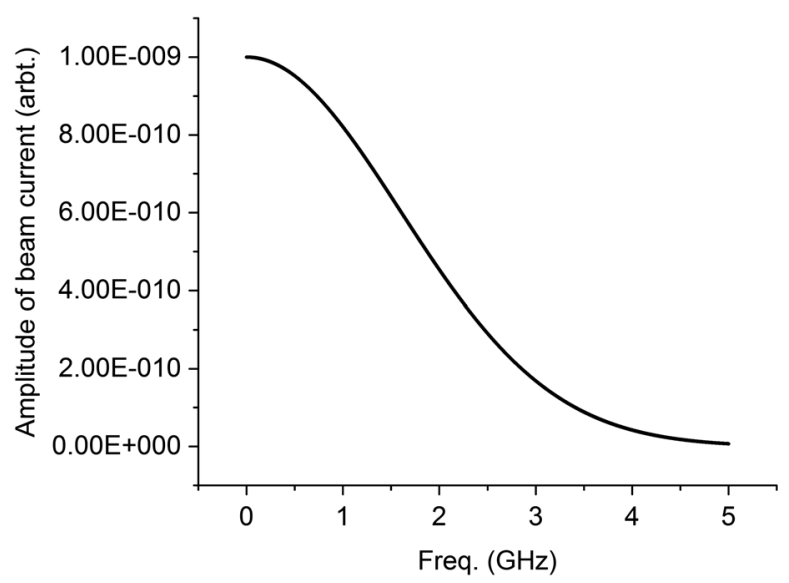

FIG. 5. Fourier transform of beam current used for the simulation of the test BPM with particle beam. 


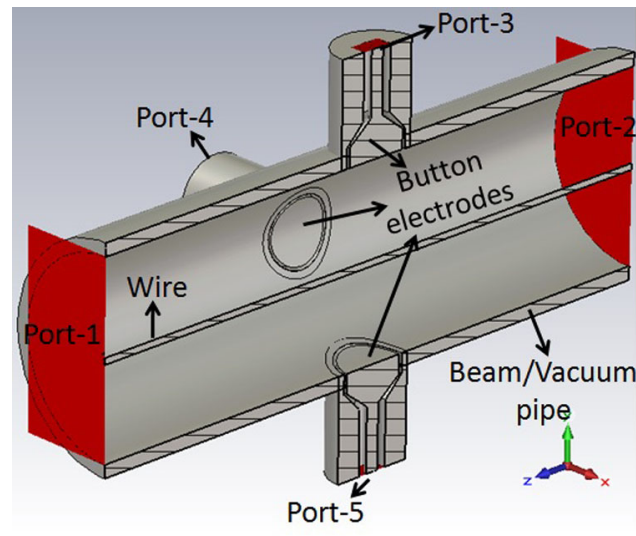

FIG. 6. CST model for the simulation of the conventional coaxial wire method used for the calculation of transfer impedance of the test BPM.

shown in Fig. 7. The only difference between the simulation model of the modified and the conventional coaxial wire methods lies in modeling of port- 1 and port-2. For the case of the $50 \Omega$ downstream port, port- 1 and port- 2 are modeled such that they act as $50 \Omega$ waveguide ports. Whereas, for the case of the shorted downstream port, only port- 1 is modeled. The results are discussed in the next subsection.

\section{Transfer impedance of the test BPM}

The S-parameters $\left(S_{21}\right.$ and $S_{i 1} ; i=3$ to 6$)$ obtained through the transient solver of the CST MICROWAVE STUDIO are shown in Fig. 8. For the conventional wire method, the transfer impedance is calculated using Eq. (1) and for the modified wire method, the transfer impedance is calculated using Eqs. (18) and (20). The results are shown in Fig. 9. For the modified coaxial wire method having a shorted downstream port, the denominator of Eq. (20) is zero, but the simulated value of the $\mathrm{S}$-parameter is finite at $3 \mathrm{GHz}$.

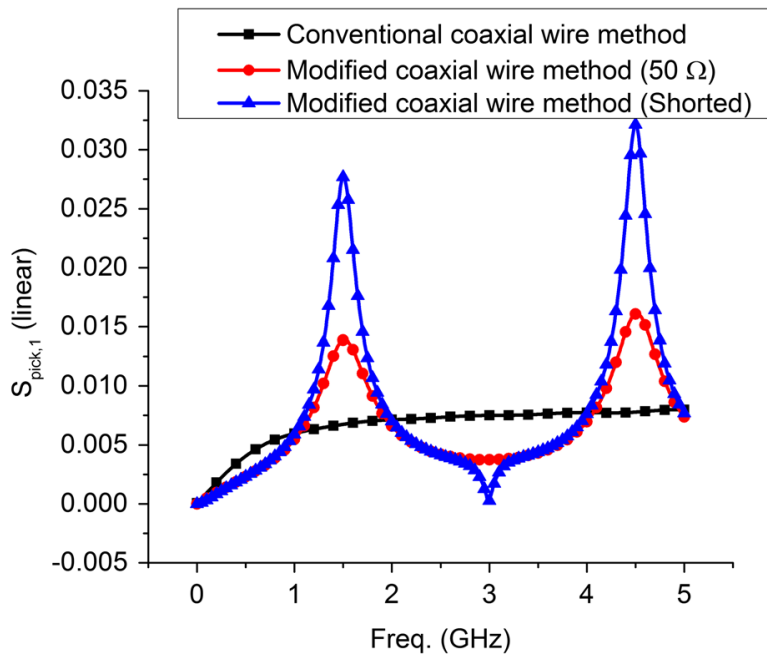

FIG. 8. S-parameters obtained through simulation of conventional and modified coaxial wire methods for the calculation of transfer impedance of the test BPM.

Therefore, the transfer impedance shows discontinuity at $3 \mathrm{GHz}$. In general, for setup with the shorted end, the denominator of Eq. (20) becomes zero at frequency $f=N_{c} /(L+\Delta L)$. Here $N=0,1,2, \ldots$ and c is velocity of the light. Therefore, to avoid singularity in the measurement, one should avoid this frequency or the length " $L$ " should be chosen (using extended beam pipe) in such a way that there is no singularity at desired frequency or frequency range.

The simulation results also show that, for the modified coaxial wire method, the S-parameters have resonances at 1.5 and $4.5 \mathrm{GHz}$. These resonances are due to the fact that the coaxial structure forms a cavity of length $L=100 \mathrm{~mm}$. Thus, the structure has discrete resonances (trapped modes) at $L=(2 n-1) \lambda / 2(n=1,2,3, \ldots)$ with $\lambda$ being wavelength at resonance. The electromagnetic field corresponding to these resonances (trapped modes) decays through the coaxial
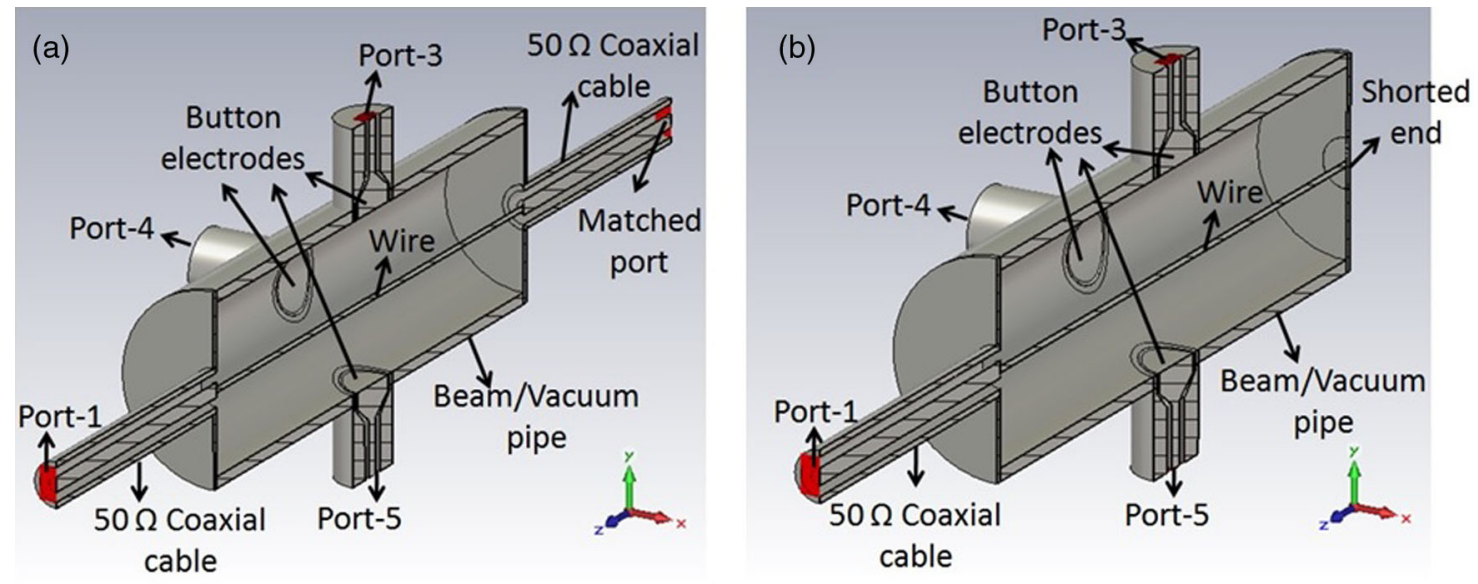

FIG. 7. CST model for the simulation of the modified coaxial wire method used for the calculation of transfer impedance of the test BPM. (a) $50 \Omega$ downstream port and (b) shorted downstream port. 


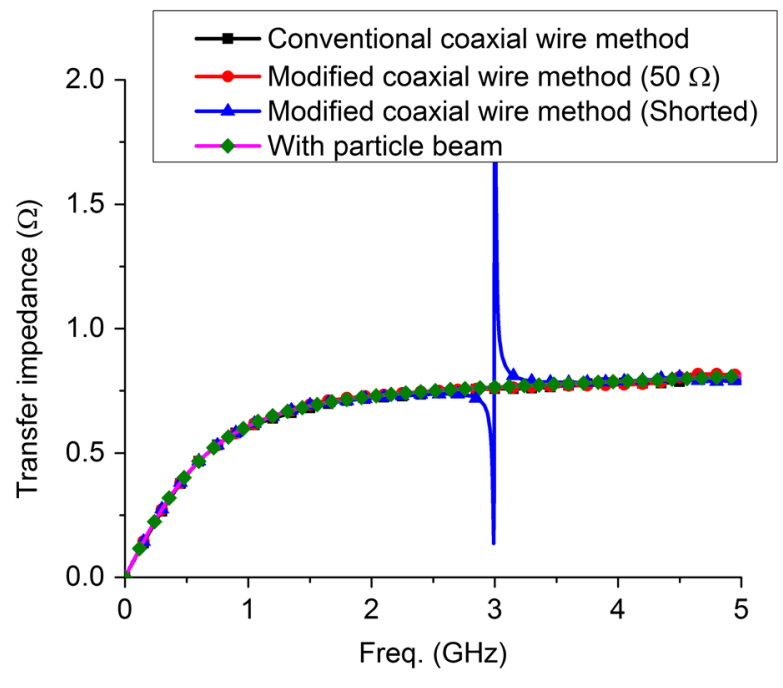

FIG. 9. Transfer impedance of the test BPM obtained through simulation of the BPM.

wire. The rate of decay of the electromagnetic field depends on the mismatch between DUT and the external circuit and it decreases with increasing impedance mismatch. Equations (18) and (20) have been derived by superposition of infinite numbers of reflections. Therefore, mathematically, these resonances have no effect on the validity of Eqs. (18) and (20) for the calculation of transfer impedance using the modified coaxial wire method as indicated in Fig. 9.

\section{EXPERIMENTAL VERIFICATION}

The modified coaxial method is applied to measure transfer impedance of IDBPI and the results are compared with simulations. The geometry of IDBPI is shown in Fig. 10. The vacuum chamber of IDBPI has a racetrack profile having vertical aperture $17 \mathrm{~mm}$ and horizontal aperture $81 \mathrm{~mm}$. The IDBPI consists of four pickup

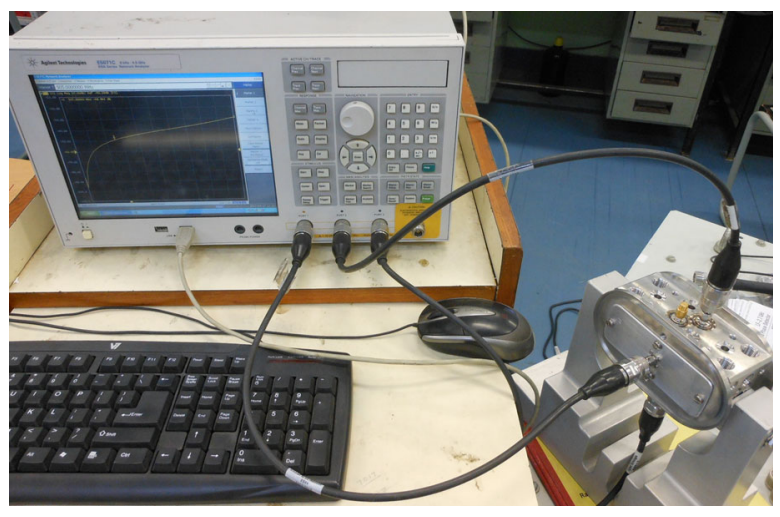

FIG. 11. Modified coaxial wire setup for the measurement of transfer impedance of IDBPI.

electrodes. The radius of pickup electrode is $4.5 \mathrm{~mm}$ and the annular gap between pickup electrode and the BPM body is $1 \mathrm{~mm}$. There is one pickup electrode in each quadrant of the transverse plane. The pickup electrodes in the first and third quadrants have longitudinal separation of $16 \mathrm{~mm}$ with respect to the pickup electrodes in the second and fourth quadrants. The horizontal separation between pickup electrodes is $12 \mathrm{~mm}$. Details of the IDBPI design are given in Refs. [14-15].

\section{A. Measurement}

The modified coaxial wire method having the shorted downstream port has been implemented to measure transfer impedance of the IDBPI. The measurement setup is shown in Fig. 11. Two aluminum plates each having thickness $2 \mathrm{~mm}$ are mounted at the end of the IDBPI body. A SubMiniature version A (SMA)-type electrical feedthrough is mounted at the center of one aluminum plate such that the central pin of the SMA feedthrough protrudes inside and its connector part lies outside of the IDBPI. A copper wire is stretched from the pin of the electrical feedthrough and
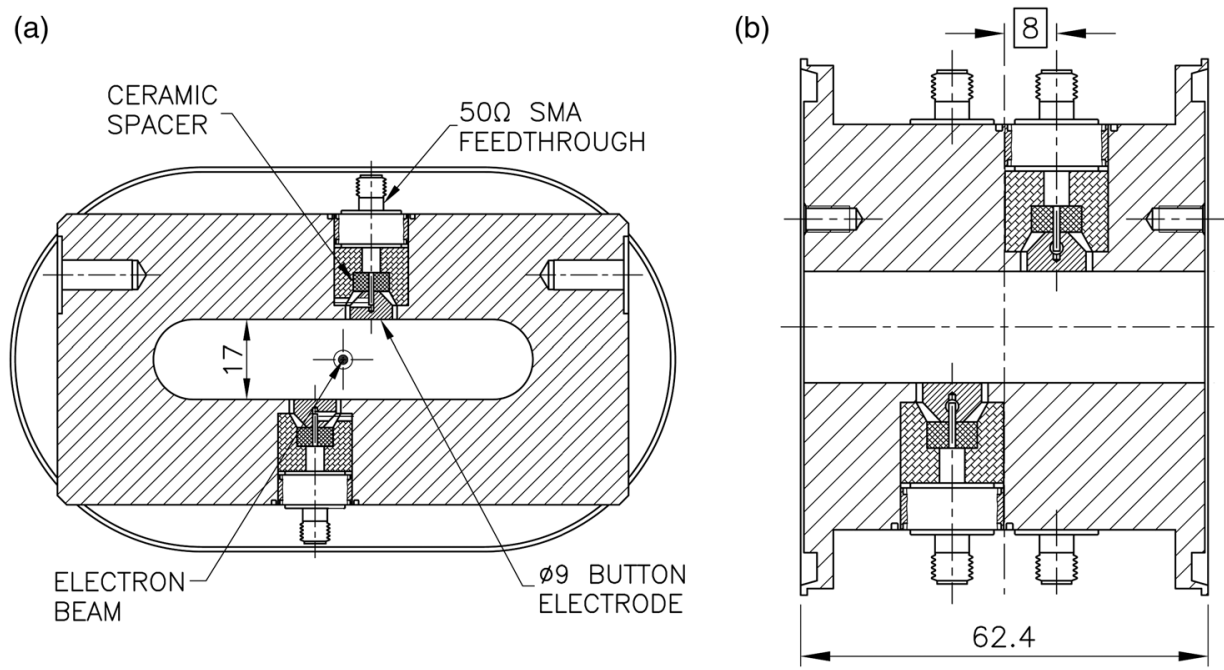

FIG. 10. Cross sectional view of IDBPI. (a) Front view and (b) side view. Unit of dimensions shown in the above figure are in mm. 

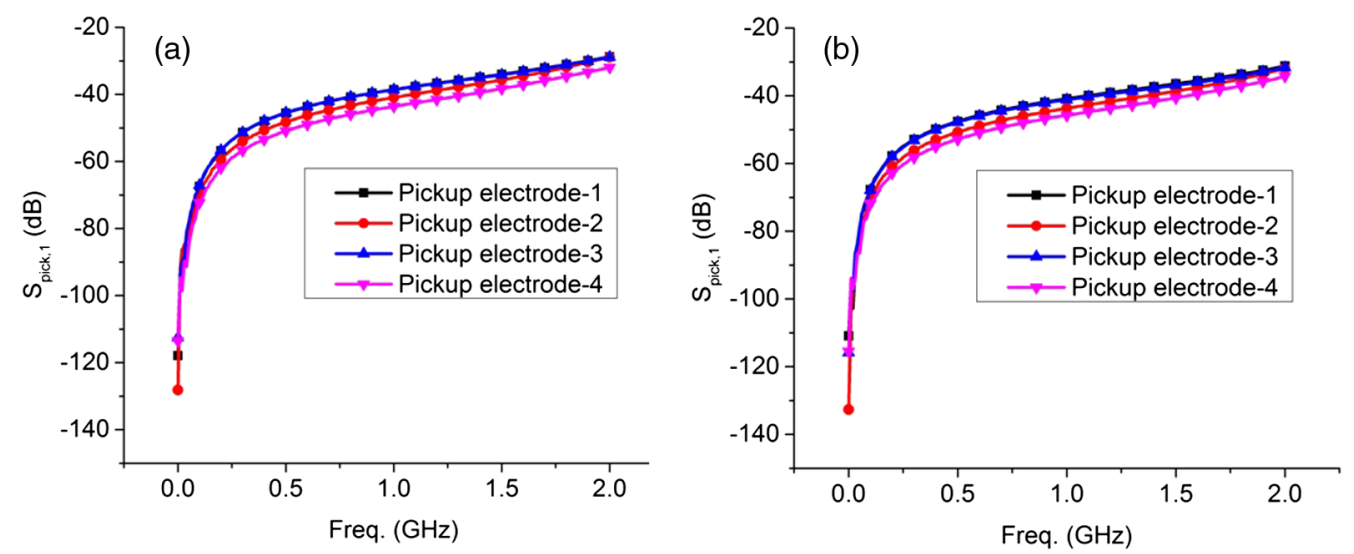

FIG. 12. S-parameters $\left(\mathrm{S}_{\text {pick, } 1}\right)$ of IDBPI measured using the modified coaxial wire method. (a) $1.5 \mathrm{~mm}$ wire diameter and (b) $0.6 \mathrm{~mm}$ wire diameter.

fixed (electrically shorted) at the center of the other aluminum plate.

Port-1 of the VNA is connected to the coaxial wire and S-parameters $\left(S_{\text {pick, } 1}\right)$ from the coaxial wire to the pickup electrodes are measured one by one using port- 2 of the VNA. While measuring S-parameter for a given electrode, the other electrodes are terminated with load of $50 \Omega$ each. Measurements are done using two different wires having diameter 1.5 and $0.6 \mathrm{~mm}$. The measured S-parameters for different pickup electrodes are shown in Fig. 12. The pickup electrodes of IDBPI have longitudinal shift from the center of the BPM. Therefore, pickup electrodes which are close to the input port are labeled as electrode $1 \& 3$ and pickup electrodes which are far from the input port are called electrode $2 \& 4$.

For racetrack geometry, having vertical aperture much smaller than horizontal aperture, the characteristic impedance of coaxial structure is given by [12]

$$
Z_{w} \approx 60 \ln \left(1.27 \frac{H}{D_{w}}\right)
$$

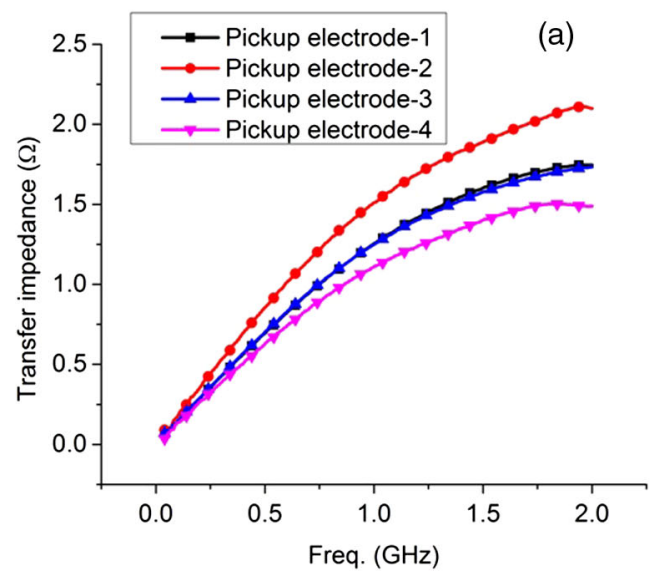

In Eq. (21), $D_{w}$ is wire diameter and $H$ is vertical aperture. For $H=17 \mathrm{~mm}$, the characteristic impedance of the coaxial structure formed by wires of diameter 1.5 and $0.6 \mathrm{~mm}$ comes out to be $\sim 161.7$ and $\sim 215 \Omega$, respectively. The reflection coefficient ' $|\rho|$ ' at plane-1 is given by

$$
|\rho|=\frac{\left|Z_{w}-Z_{0}\right|}{\left|Z_{w}+Z_{0}\right|}
$$

Using $Z_{w}=161.7$ and $\sim 215 \Omega$ in Eq. (22), the reflection coefficients come out to be $\sim 0.528$ and $\sim 0.623$ for 1.5 and $0.6 \mathrm{~mm}$ wire diameters, respectively. The transfer impedance of different pickup electrodes, calculated from measured S-parameters and Eq. (20), are shown in Fig. 13.

As indicated in Fig. 13, the transfer impedances calculated experimentally for different pickup electrodes vary from each other. The transfer impedance of electrode- 1 and electrode- 3 is very close to the expected value whereas it is relatively higher for electrode- 2 and lower for electrode- 4 . The possible reason for this could be that the wire has slight offset or angle with respect to the axis of the IDBPI.

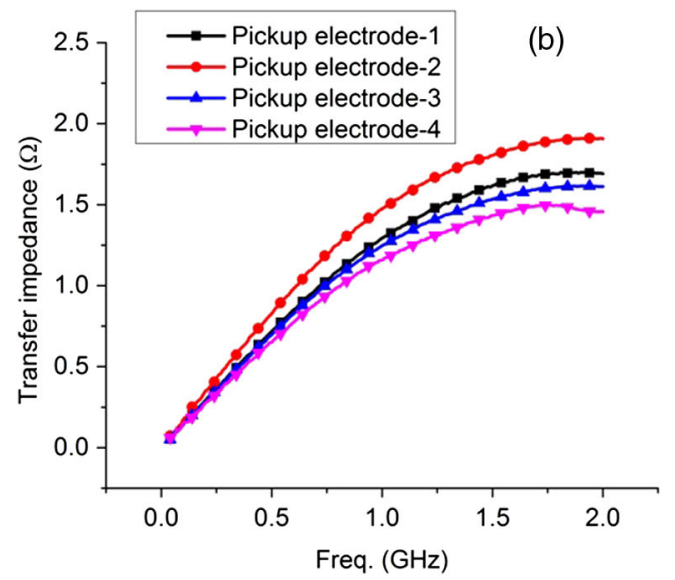

FIG. 13. Transfer impedance of different electrodes of IDBPI measured using modified coaxial wire method. (a) 1.5 mm wire diameter and (b) $0.6 \mathrm{~mm}$ wire diameter. 


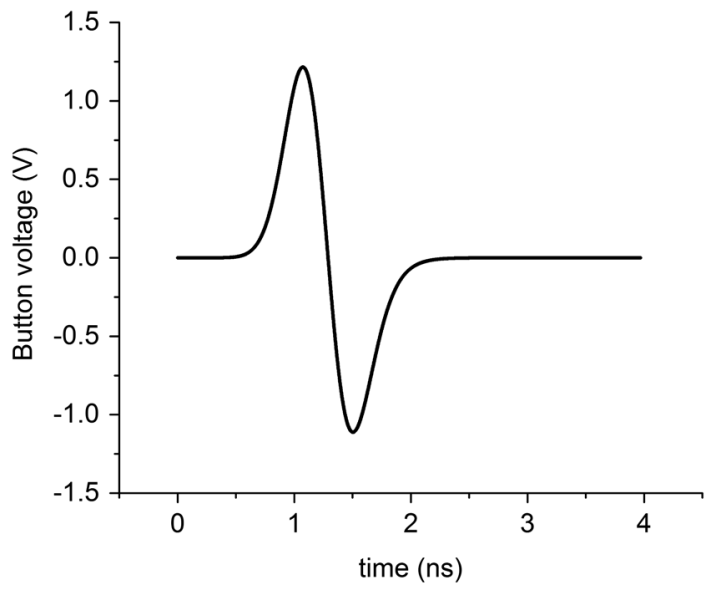

FIG. 14. Voltage signal induced at the button electrode of the IDBPI obtained through simulation with particle beam of rms length $60 \mathrm{~mm}$.

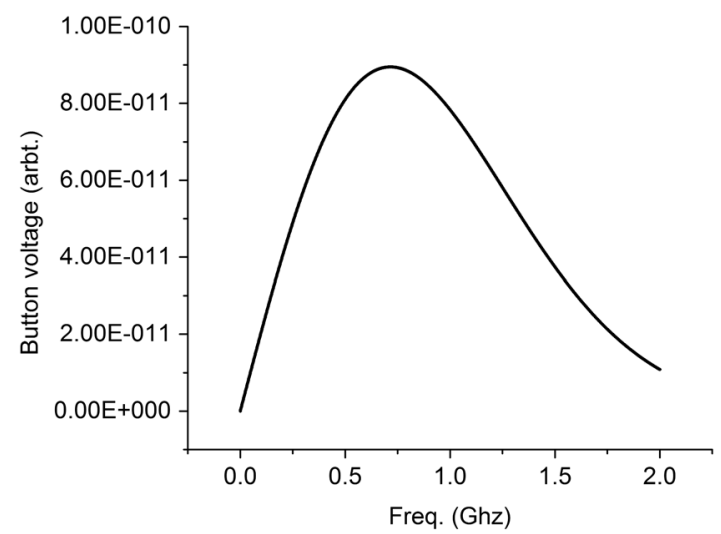

FIG. 15. Fourier transform of the voltage signal induced at the button electrode of the IDBPI obtained through simulation with particle beam of rms length $60 \mathrm{~mm}$.

The position sensitivity of the IDBPI is very high $\left(\sim 0.125 \mathrm{~mm}^{-1}\right)$, therefore, even a slight offset of wire can change the output signal significantly. The longitudinal distance of electrodes 2 and 4 from the electrodes 1 and 3 is
$16 \mathrm{~mm}$. Therefore, for example, an angle of $\sim 3.6^{0}$ gives an offset of $\sim 1 \mathrm{~mm}$ at the location of electrodes 2 and 4 as compared to electrodes 1 and 3 . This can change the output (transfer impedance) by $\sim 12.5 \%$. The transfer impedance corresponding to the axis of the IDBPI is calculated by averaging the transfer impedance of all pickup electrodes and presented in Sec. IV B.

\section{B. Measurement versus simulation}

This section presents a comparison of measured results with simulations performed using CST STUDIO SUITE. The $\mathrm{S}$-parameters and transfer impedance of the IDBPI is obtained by simulating it with particle beam, the conventional coaxial wire method and the modified coaxial wire method as explained in Sec. III. To directly compare the simulated S-parameters with the measured data, the diameter of the wire is taken to be equal to that used in measurement. The button signal and S-parameters obtained through simulations are shown in Figs. 14-16.

Comparison of S-parameters obtained through simulation of the modified coaxial wire method using CST MICROWAVE STUDIO (CST MWS) with measured data is shown in Fig. 17. The difference in S-parameters (in $\mathrm{dB}$ ) of different electrodes is very small as compared to the complete range of the S-parameters. Therefore, the linear scale has been used for the comparison of S-parameters of different electrodes obtained through simulation and measurement.

The transfer impedances (along the axis of the IDBPI) obtained through different simulations and measurement are shown in Fig. 18. Up to $\sim 1.8 \mathrm{GHz}$, there is very good agreement between transfer impedance obtained through bench measurement and simulations. Above $1.8 \mathrm{GHz}$, the results of bench measurement show slight deviation from the simulations. This deviation is due to the resonance at $\sim 2.4 \mathrm{GHz}$. As we approach this resonance, the measured results become quite sensitive to the length of DUT (L) and offset $(\Delta L)$ of the electrodes from the center of the DUT. The dependency of the measured results on the accuracies

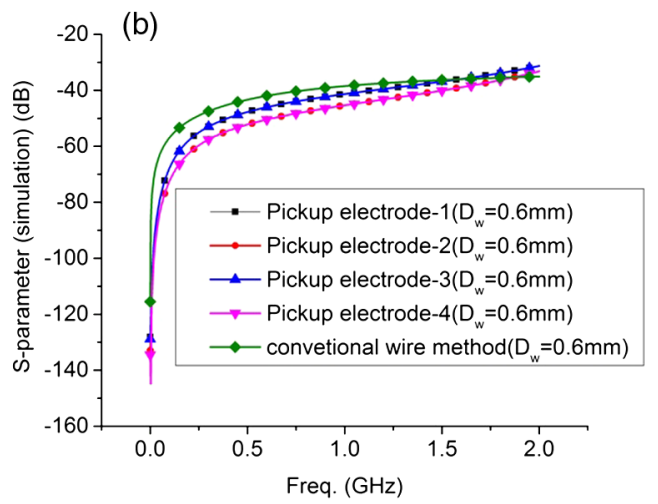

FIG. 16. S-parameters obtained through simulation of conventional and modified (pickup electrode 1 to 4 ) coaxial wire methods for the calculation of transfer impedance of IDBPI. (a) $1.5 \mathrm{~mm}$ wire diameter and (b) $0.6 \mathrm{~mm}$ wire diameter. 

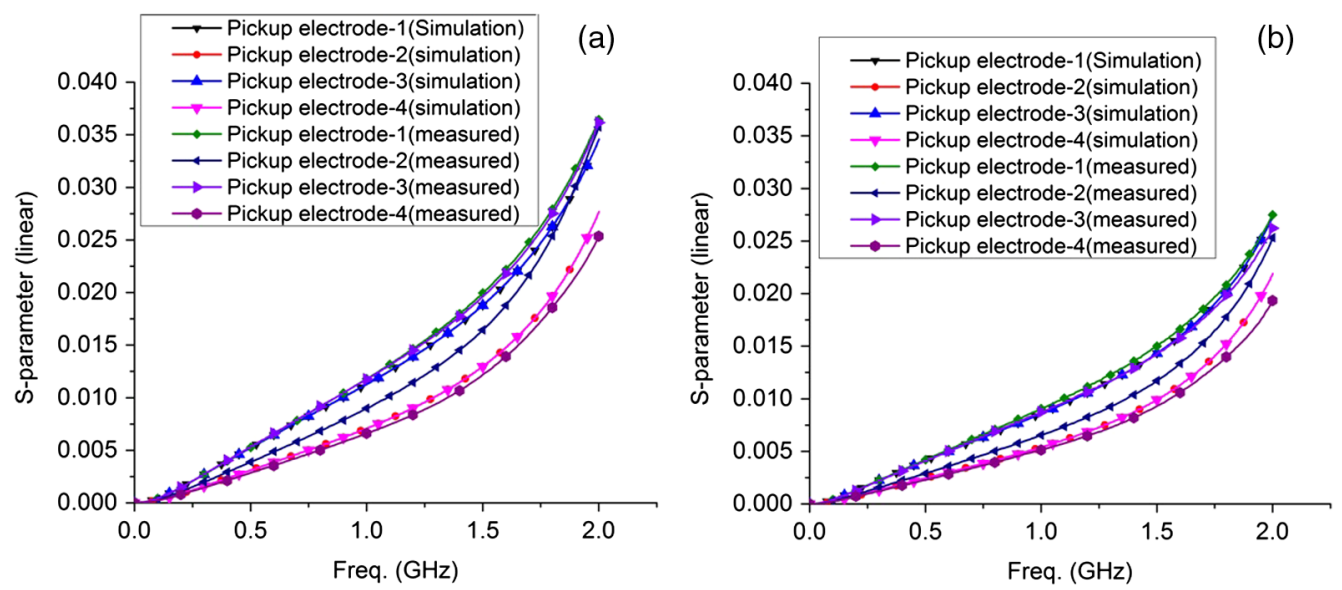

FIG. 17. Comparison of S-parameters of IDBPI obtained through simulation with measured data for different electrodes. (a) 1.5 mm wire diameter and (b) $0.6 \mathrm{~mm}$ wire diameter.

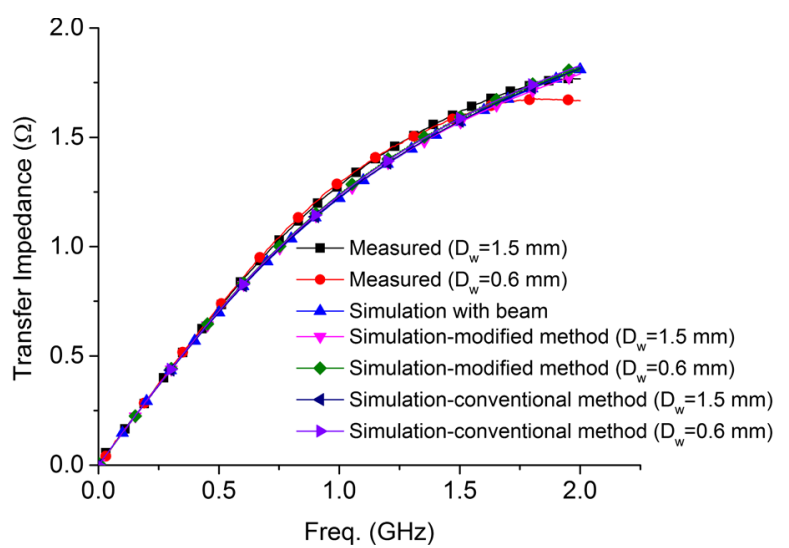

FIG. 18. Comparison of the transfer impedance of IDBPI obtained experimentally with simulations.

of $\mathrm{L}$ and $\Delta L$ is significant only near resonance and in the rest of the region (below $1.8 \mathrm{GHz}$ ) measured results are not much sensitive to these parameters. The accuracy of transfer impedance calculated using Eq. (20) depends on the accuracy of the different parameters like measured S-parameters, length of DUT (L) and longitudinal offset $(\Delta L)$. Therefore, near resonance, the accuracy of the measured results can be improved by increasing the accuracy of parameters used in Eq. (20).

\section{SUMMARY AND CONCLUSION}

Measurement of the transfer impedance is very important for the characterization of the BPM. The conventional coaxial wire method is a standard technique to measure transfer impedance of the BPM. The conventional coaxial wire method requires impedance matching between external circuit and DUT (BPM). Generally, the implementation of impedance matching is done using impedance matching electrical networks or long tapered physical structures. In the conventional wire method, if characteristics impedance of the coaxial structure changes, one has to redesign/ replace existing impedance matching elements (electrical network or tapered structure) accordingly.

In this paper, we have presented the modified coaxial wire method for the bench measurement of the transfer impedance of capacitive pickups like button electrodes and shoe box BPMs. The modified coaxial wire method is easy to implement (no impedance matching is required) and immune to any change in impedance of coaxial structure arising due to change in wire diameter or any other factor. In the modified coaxial wire method, the impedance matching elements have been eliminated. The effect of impedance mismatch has been solved mathematically and new expressions for the transfer impedance have been derived.

The proposed method is validated through simulation of a test BPM using different methods in CST STUDIO SUITE. The modified coaxial method is also applied to measure transfer impedance of the insertion device beam position indicator (IDBPI) developed for Indus-2. The results obtained using the modified coaxial wire method showed close agreement with the results obtained through simulation using CST STUDIO SUITE.

Close agreement between measured and simulated results suggests that the modified coaxial method can be used to measure transfer impedance of capacitive BPMs like button electrodes and shoe box BPM. The idea of the modified coaxial wire method can also be extended to derive equations for devices like striplines which have directional properties. In such a case, where signal depends on direction of the beam, one has to consider the polarity of the output signals due to multiple reflections inside the device. Therefore, the proposed method can also be useful for the calibration of stripline pickup/kickers.

The coaxial structure in the modified coaxial wire method forms a cavity of length $\mathrm{L}$. Thus, the structure has discrete resonances at $L=(2 n-1) \lambda / 2(n=1,2,3, \ldots)$ with $\lambda$ being wavelength at resonance. Near these resonances, the calculation of transfer impedance becomes 
quite sensitive to the parameters of coaxial structure and measured S-parameters. Therefore, the modified coaxial wire method should be used with care near resonance in the structure.

[1] M. Sands and J. Rees, A bench measurement of the energy loss of a stored beam to a cavity, SLAC Report No. PEP95, 1974.

[2] F. Caspers, Beam impedance measurements using the coaxial wire method, CERN Report No. PS/88-59 (AR/ OP).

[3] G. Di Massa and M. Rosaria Masullo, Beam Impedance Measurement Coaxial Wire Method, in Proceedings of the 1991 Particle Accelerator Conference, San Francisco, CA, 1991 (IEEE, New York, 1991).

[4] J. J. Song, Experimental study of beam coupling impedance, Part-1: Longitudinal impedance measurement techniques, LS Note-201, APS-ANL, 1991.

[5] L. S. Walling, D. E. McMurry, D. V. Neuffer, and H. A. Thiessen, Transmission line impedance measurements for an advanced hadron facility, Nucl. Instrum. Methods Phys. Res., Sect. A 281, 433 (1989).

[6] L. Y. Dong, H. L. Sheng, and W. Sheng, Coupling impedance measurements of the extractor kicker in CSNS/RCS, in Proceedings of the Symposium on Accelerator Physics, Lanzhou, China (2014), pp. 102-105, http://accelconf.web .cern.ch/AccelConf/SAP2014/papers/thamh4.pdf.

[7] H. Hahn and F. Pedersen, On coaxial wire measurements of the longitudinal coupling impedance, BNL 50870, 1978.

[8] M. J. barnes, F. Caspers, T. Kroyer, E. Mitral, F. Roncarolo, and B. Salvant, Measurement of transverse impedance of licker magnets using the coaxial wire method, in Proceedings of the 23rd Particle Accelerator Conference,
Vancouver, Canada, 2009 (IEEE, Piscataway, NJ, 2009), pp. 1726-1728.

[9] M. R. Masullo, V. G. Vaccaro, and M. Panniello, The stretched wire method: A comparative analysis performed by means of the mode matching technique, in Proceedings of the 25th International Linear Accelerator Conference, LINAC-2010, Tsukuba, Japan (KEK, Tsukuba, Japan, 2010).

[10] J. N. Corlett, Coaxial wire impedance measurement of BPM buttons for the PEP-II B-Factory, Lawrence Berkeley National Laboratory, Report No. LBL-37782, 1995, https://escholarship.org/uc/item/9d49k20v.

[11] F. Marcellini, M. Serio, A. Stella, and M Zobov, DA $\varphi$ NE broad-band button electrodes, Nucl. Instrum. Methods Phys. Res., Sect. A 402, 27 (1998).

[12] T. Kroyer, F. Caspers, and E. Gaxiola, Longitudinal and transverse wire measurements for the evaluation of impedance reduction measures on the MKE extraction kickers, Report No. CERN-AB-Note-2007-028, 2007.

[13] CST STUDIO SUITE website: http://www.cst.com.

[14] Mukesh Kumar, L. K. Babbar, S. Yadav, T. A. Puntambekar, and C. P. Navathe, Physics design of beam position indicator for insertion device section in Indus-2, in Proceedings of InPAC-2013 (VECC, Kolkata, India, 2013).

[15] L. K. Babbar, M. Kumar, D. P. Yadav, B. N. Upadhyaya, R. sridhar, and T. A. Puntambekar, Mechanical design, development and installation of ultra high vacuum compatible beam position indicators for insertion devices in Indus-2, in Proceedings of InPAC-2015 (TIFR, Mumbai, India, 2015).

[16] http://www.cat.gov.in/technology/accel/indus/indus2.html.

[17] M. Kumar, L. K. Babbar, A. C. Holikatti, S. Yadav, Y. Tyagi, T. A. puntambekar, and V. K. Senecha, Improved design and in situ measurements of new beam position monitors for Indus-2, J. Instrum. 13, P01003 (2018). 